\title{
Article \\ Application of Fourier Sine Transform to Carbon Nanotubes Suspended in Ethylene Glycol for the Enhancement of Heat Transfer
}

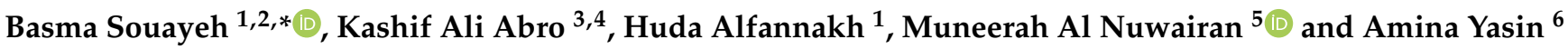 \\ 1 Department of Physics, College of Science, King Faisal University, P.O. Box 400, Al-Ahsa 31982, Saudi Arabia; \\ halfannakh@kfu.edu.sa \\ 2 Laboratory of Fluid Mechanics, Physics Department, Faculty of Science of Tunis, University of Tunis EI Manar, \\ Tunis 2092, Tunisia \\ 3 Faculty of Natural and Agricultural Sciences, Institute of Ground Water Studies, University of the Free State, \\ Bloemfontein 9300, South Africa; kashif.abro@faculty.muet.edu.pk \\ 4 Department of Basic Sciences and Related Studies, Mehran University of Engineering and Technology, \\ Jamshoro 67480, Pakistan \\ 5 Department of Mathematics and Statistics, College of Science, King Faisal University, P.O. Box 400, \\ Al-Ahsa 31982, Saudi Arabia; msalnuwairan@kfu.edu.sa \\ 6 Preparatory Year Deanship, Department of Basic Sciences, King Faisal University, P.O. Box 400, \\ Al Hofuf 31982, Saudi Arabia; ayasin@kfu.edu.sa \\ * Correspondence: bsouayeh@kfu.edu.sa
}

check for updates

Citation: Souayeh, B.; Ali Abro, K.; Alfannakh, H.; Al Nuwairan, M.; Yasin, A. Application of Fourier Sine Transform to Carbon Nanotubes

Suspended in Ethylene Glycol for the Enhancement of Heat Transfer. Energies 2022, 15, 1200. https:// doi.org/10.3390/en15031200

Academic Editor: Gabriela Huminic

Received: 24 November 2021

Accepted: 10 December 2021

Published: 7 February 2022

Publisher's Note: MDPI stays neutral with regard to jurisdictional claims in published maps and institutional affiliations.

Copyright: (C) 2022 by the authors. Licensee MDPI, Basel, Switzerland. This article is an open access article distributed under the terms and conditions of the Creative Commons Attribution (CC BY) license (https:// creativecommons.org/licenses/by/ $4.0 /$ )

\begin{abstract}
There is no denying fact that nanoparticles of carbon nanotubes are employed to improve the performance of thermal stability in comparison with traditional nanoparticles, this is because nanoparticles of carbon nanotubes possess outstanding material properties. In this manuscript, a mathematical model of mixed convective flow based on carbon nanotubes suspended in ethylene glycol has been developed and derived by means of Fourier Sine transform. In order to analyze the thermophysical properties of nanofluid, the temperature and velocity profiles have been investigated through fractional derivative and integral transforms. The comparative analysis of single and multiwalled carbon nanotubes has been presented for the sake of enhancement of heat transfer. It is worth mentioning that embedded rheological parameters have shown the sensitivity for the enhancement of heat transfer with and without fractional techniques through graphical illustration.
\end{abstract}

Keywords: nanoparticles of carbon nanotubes; integral transforms; rate of heat transfer; high sensitivity of rheological parameters

\section{Introduction}

The carbon nanotubes have become high-strength and lightweight materials due to their consideration as the structural material, the strongest material and stiffest material in a broad range of research fields. In fact, carbon nanotubes can come in a variety of impressive electrical as well as thermal properties, sizes, hollow cylinders of graphene, and shapes through which one can control the temperature of the deposition processes [1-5]. In this connection, Halelfadl et al. [6] investigated the experimental rheology of carbon nanotubes in water-based nanofluids stabilized by a surfactant for a large aspect-ratio. Here, the viscosity of both nanofluid and base-fluid (deionized-water) were affected by temperature distribution. Additionally, the relative viscosity perceived to be dependent on temperature at high shear rate by Maron-Pierce equation. The thermal and physical properties of carbon nanotubes' nanofluids with water as a base fluid stabilized by Halelfadi et al. [7]. It was observed that temperature failed to be function of density as well as relative density while increase in particle volume fraction resulted affective thermal conductivity and relative viscosity. Kandasamy et al. [8] studied the impact of chemical reaction on convective flow of single wall carbon-nanotubes (SWCNTs) water-based nanofluid with alumina and copper 
over a horizontal plate under slip condition. They solved the coupled nonlinear governing equations numerically. The results represented that the thickness of diffusion boundary layer for $\mathrm{Cu}$-water is stronger than Alumina-water single wall carbon-nanotubes nanofluid with increasing chemical reaction. Mustafa and Junaid [9] solved the magnetohydrodynamics (MHD) Casson nanofluid flow embedded by non-linear stretching sheet by using RK-fourth and fifth order numerical method with shooting technique. They analyzed that temperature as well as volume fraction of nanoparticle decrease thermal efficiencies. The single and multi-walled carbon nanotubes have been inspected by Kashif and Ilyas [10] for thermal performance by invoking non-singular and non-local kernels from modern fractional derivatives. This study emphasized the magnetized and non-magnetized behavior of single and multi-walled carbon nanotubes. Kandasamy et al. [11] investigated the heat transfer flow of magnetized single wall CNTs-water and $\mathrm{Cu}$-water nanofluids under the influence of solar radiation past a porous wedge-sheet. Here, the influence of magnetic parameter, radiation parameter and thermal stratification is inspected on velocity and temperature distributions. The thermal effects of SWCNTs-water with $\mathrm{Cu}$-water have been compared graphically and referred new aspects. Khan et al. [12] explored the critical study for convection flow of water-based carbon-nanotube nanofluid in vertical channel via modern non-singular and non-local fractional derivative based on kernel. The fractionalized governing equation have been solved by new type of mathematical techniques so called Zakian's algorithm for Laplace inversion. The final solution is compared with two existing numerical methods via Laplace inversion for the sake of good agreement with existing numerical techniques.

In this continuity, the recent studies for the thermal performance can be traced out here with thermal study with fractional derivatives [13-17], thermal study with Laplace and Fourier sine transforms [18-20], thermal study with classical approaches [21-23] and thermal study with analytical and numerical techniques [24-36]. Motivated by the above discussion on the thermal assumptions, a mathematical model of mixed convective flow based on carbon nanotubes suspended in ethylene glycol has been developed and derived by means of Fourier Sine transform. In order to analyze the thermophysical properties of nanofluid, the temperature and velocity profiles have been investigated through fractional derivative and integral transforms. The comparative analysis of single and multi-walled carbon nanotubes has been presented for the sake of enhancement of heat transfer. It is worth mentioning that embedded rheological parameters have shown the higher sensitivity for the enhancement of heat transfer with and without fractional techniques through graphical illustration. This manuscript is organized as follows as: in Section 2, setting of the problem via fractional modeling based on carbon nanotubes of single and multiwall is discussed; In Section 3, the non-singular kernel-based technique is utilized to solve the dynamic equations of mixed convection flow of nanofluid functionalized by carbon nanotubes; In Section 4, the results with parametric discussion and conclusion are presented.

\section{Setting of the Problem via Fractional Modeling Based on Carbon Nanotubes}

Although different types of carbon nanotubes (CNTs) for instance zigzag carbon nanotubes, armchair carbon nanotubes, and chiral carbon nanotubes are employed for various applications yet single and multi-walled carbon nanotubes have been used for decades in battery, fuel cells and electrochemical applications. Under the assumption of isothermal temperature and porosity, mixed convection flow of nanofluid based on ethylene glycol over an inclined vertical plate is subjected to carbon nanotubes of single and multi-wall. Due to very small Reynolds number, electrically conducting nanofluid can induce magnetic field due to polarization. At the initial stage, the system is in the static position, because $t \leq 0$. If $t=0^{+}$, the inclined plate produces oscillations on the basis of 
$U_{0} H(t) \cos (\omega t)$. Due to this fact, the ambient temperature of the plate $T_{\infty}$ rises to $T_{\infty}$ as sketched in Figure 1. The Casson fluid model is developed for enhanced heat transfer as [28]:

$$
\begin{aligned}
& \left(\rho_{n f} \frac{\partial}{\partial t}+\frac{\mu_{n f} \varphi}{k}\left(1+\frac{1}{\beta_{0}}\right)+\sigma_{n f} B_{0}^{2} \sin \gamma\right) u(y, t)=\mu_{n f}\left(1+\frac{1}{\beta_{0}}\right) \frac{\partial^{2} u(y, t)}{\partial y^{2}} \\
& +\left(\rho \beta_{T}\right)_{n f} g \cos \delta\left(T(y, t)-T_{\infty}\right), \quad\left(\rho C_{p}\right)_{n f} \frac{\partial T(y, t)}{\partial t}=k_{n f} \frac{\partial^{2} T(y, t)}{\partial y^{2}}
\end{aligned}
$$

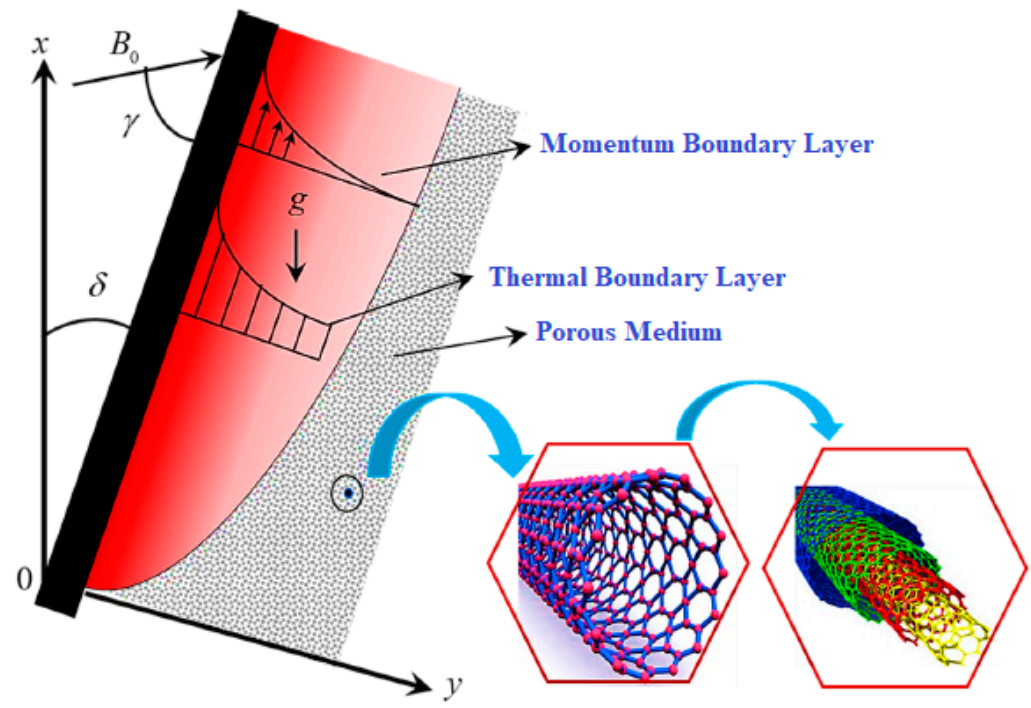

Figure 1. Schematic view of the physical setup.

Equation (1) is momentum and energy equations respectively, such equations have been derived by invoking Fourier law of heat conduction, Darcy's law and Boussinesq approximation. The Equation (1) is subjected to the following initial and boundary conditions with bounded domain $y \geq 0$ and $t>0$ as written in Equation (2):

$$
\begin{aligned}
& u(y, 0)=0, u(0, t)=U_{0} \cos \omega t, u(y, 0) \rightarrow \infty \\
& T(y, 0)=T_{\infty}, T(0, t)=T_{w}, T(y, 0) \rightarrow \infty
\end{aligned}
$$

The functional parameters and thermophysical properties of nanofluid are described in Appendix A (Equations (A1)-(A6)). Employing Appendix A Equation (A7) in Equations (1) and (2), the governing equations of Casson fluid without fractional derivative is developed for enhanced heat transfer as:

$$
\begin{gathered}
\left(\frac{\partial}{\partial t}+\frac{\mathfrak{B}_{2} M \sin \gamma}{\mathfrak{B}_{0}}+\frac{\mathfrak{B}_{3}}{\mathfrak{B}_{0} K}-\frac{\mathfrak{B}_{1}}{\mathfrak{B}_{0} \beta} \frac{\partial^{2}}{\partial y^{2}}\right) u(y, t)=\frac{\mathfrak{B}_{4}}{\mathfrak{B}_{0}} G r \theta(y, t) \cos \delta, \\
\left(\frac{\partial}{\partial t}-\frac{\mathfrak{B}_{6}}{\operatorname{Pr} \mathfrak{B}_{5}} \frac{\partial^{2}}{\partial y^{2}}\right) \theta(y, t)=0
\end{gathered}
$$

While, imposed conditions are:

$$
\begin{aligned}
& u(y, 0)=0, u(0, t)=\cos \omega t, u(y, \infty)=0 \\
& T(y, 0)=0, T(0, t)=1, T(y, \infty)=0
\end{aligned}
$$

Equations (3) and (4) are the non-fractional governing equation. In order to transform non-fractional governing equation into fractionalized governing equation, the AtanganaBaleanu time-fractional differential operator [29,30] is invoked on Equations (3) and (4), we arrive at:

$$
\left(\text { Atangana-Baleanu }_{\mathfrak{D}_{t}^{\mathfrak{q}}}+\frac{\mathfrak{B}_{2} M \sin \gamma}{\mathfrak{B}_{0}}+\frac{\mathfrak{B}_{3}}{\mathfrak{B}_{0} K}-\frac{\mathfrak{B}_{1}}{\mathfrak{B}_{0} \beta} \frac{\partial^{2}}{\partial y^{2}}\right) u(y, t)=\frac{\mathfrak{B}_{4}}{\mathfrak{B}_{0}} G r \theta(y, t) \cos \delta,
$$




$$
\left(\text { Atangana-Baleanu }_{\mathfrak{D}_{t}^{\mathcal{q}}}-\frac{\mathfrak{B}_{6}}{\operatorname{Pr} \mathfrak{B}_{5}} \frac{\partial^{2}}{\partial y^{2}}\right) \theta(y, t)=0 .
$$

Here, Atangana-Baleanu $\mathfrak{D}_{t}^{\mathcal{q}}$ is known as Atangana-Baleanu time-fractional differential operator, that is defined as [31-33]:

$$
\text { Atangana-Baleanu }_{\mathfrak{D}_{t}^{\mathcal{q}} P} P \int_{0}^{\tau} E_{\boldsymbol{q}}\left(\frac{-\boldsymbol{q}(z-t)^{\boldsymbol{q}}}{1-\boldsymbol{q}}\right) P^{\prime}(t)(1-\boldsymbol{q})^{-1} d t
$$

Additionally, Equations (6) and (7) are the fractional governing equation in terms of the Atangana-Baleanu time-fractional differential operator as defined in Equation (8), such equations can be solved by the following system flow chart of the problem as shown in Figure 2:

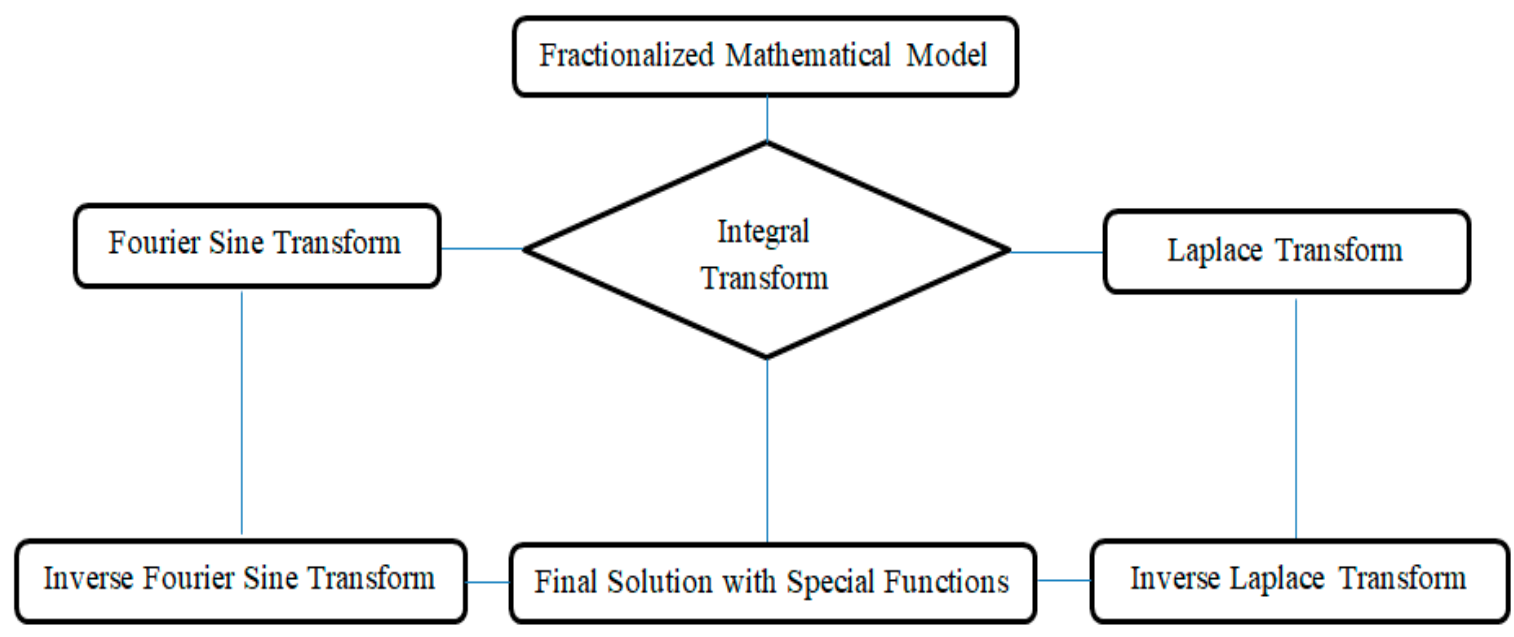

Figure 2. Flow chart for solution of the problem.

\section{Solutions via Non-Singular Kernel}

In the current research, the non-singular kernel-based technique is utilized to solve the dynamic equations of mixed convection flow of nanofluid functionalized by carbon nanotubes. This technique transforms the integer order of differentials to non-integer order of differentials. By considering this non-singular kernel-based technique, two vital investigations are sought out for the sake of thermal estimations namely energy equation and momentum equation. Whilst, integral transforms namely Laplace and Fourier sine transforms have been invoked on the governing Equations (6) and (7) of Casson fluid with fractional derivative as the mathematical application defined as:

$$
\begin{gathered}
\mathcal{L}\{f(t)\}=\int_{0}^{\infty} e^{-k t} f(t) d t=F(k), \mathcal{F}_{s}\{f(t)\}=\sqrt{\frac{2}{\pi}} \int_{0}^{\infty} \sin (k t) f(t) d t=F_{s}(k) . \\
\mathcal{L}\left\{\text { Atangana-Baleanu }_{\mathfrak{D}_{t}^{\mathcal{q}}} f(t)\right\}=\frac{s^{\boldsymbol{q}}}{(1-\boldsymbol{q}) s^{\mathcal{q}}-\boldsymbol{q}} \bar{f}(s), \quad \mathcal{F}_{s}\left\{f^{\prime \prime}(y, t)\right\}=-\xi^{2} F_{s}(\xi, t)+\xi \sqrt{\frac{2}{\pi}} f(\xi, 0) .
\end{gathered}
$$

\subsection{Use of Non-Singular Kernel for Energy Equations}

The description of linear time invariant system of differential equations of nanofluids/fluids usually depend upon the utilization of integral transforms. In this context, governing equation of energy for Casson fluid with fractional derivative is tackled via 
Integral transform. Applying Laplace and Fourier Sine transforms on Equation (9) and (10) and Equation (7), and we have:

$$
\overline{\theta_{s}}(\xi, s)=\frac{\sqrt{\frac{2}{\pi}}}{\left\{s^{\mathcal{q}} \lambda P_{r} \mathfrak{B}_{5}+\mathfrak{B}_{6} \xi^{2} s^{\mathcal{q}}+\mathfrak{B}_{6} \xi^{2} \boldsymbol{q} \lambda\right\}} \cdot \frac{\mathfrak{B}_{6}\left(s^{\mathcal{q}}+\mathfrak{q} \lambda\right) \xi}{s} .
$$

Solving Equation (11) by the letting variables defined as $\mathfrak{B}_{7}=\mathfrak{q} \lambda, \mathfrak{B}_{8}=\lambda P_{r} \mathfrak{B}_{5}+$ $\mathfrak{B}_{6} \mathfrak{\zeta}^{2}$ and $\mathfrak{B}_{9}=\mathfrak{B}_{6} \mathfrak{\zeta}^{2} \mathfrak{B}_{7}$, we arrive at:

$$
\overline{\theta_{s}}(\xi, s)=\left\{\frac{\frac{2}{\pi}}{\xi_{s}}-\frac{2}{\pi} \frac{s^{\mathcal{q}}\left(\mathfrak{B}_{8}+\xi^{2} \mathfrak{B}_{6}\right)+\mathfrak{B}_{9}+\xi^{2} \mathfrak{B}_{6} \mathfrak{B}_{7}}{\xi s\left(s^{\left.\mathcal{q}_{\mathfrak{B}_{8}}+\mathfrak{B}_{9}\right)}\right.}\right\} .
$$

By taking functional parameters $\mathfrak{B}_{10}=\frac{\mathfrak{B}_{9}+\mathfrak{\zeta}^{2} \mathfrak{B}_{6} \mathfrak{B}_{7}}{\mathfrak{B}_{8}+\tilde{\zeta}^{2} \mathfrak{B}_{6}}$ and $\mathfrak{B}_{11}=\frac{\mathfrak{B}_{9}}{\mathfrak{B}_{8}}$, Equation (12) is invoked via inverse Fourier sine transform, the resultant expression is

$$
\bar{\theta}(y, s)=\frac{1}{s}-\left(\frac{2}{\pi} \cdot \frac{\mathfrak{B}_{8}+\xi^{2} \mathfrak{B}_{9}}{\mathfrak{B}_{8} \xi}\right) \int_{0}^{\infty} \sin (y \xi)\left(\frac{s^{\mathcal{q}}+\mathfrak{B}_{10}}{s\left(s^{\mathcal{q}}+\mathfrak{B}_{11}\right)}\right) d \xi,
$$

inverting Equation (13) by Laplace transform with special functions from Appendix A (Equations (A8)-(A10)), the optimal solution of governing equation of energy for Casson fluid with fractional derivative based on non-singular kernel is investigated as:

$$
\theta(y, t)=1-\left(\frac{2}{\pi} \cdot \frac{\mathfrak{B}_{8}+\xi^{2} \mathfrak{B}_{9}}{\mathfrak{B}_{8} \xi}\right) \int_{0}^{\infty} \sin (y \xi) \int_{0}^{t}\left\{\frac{\left(1-E_{\alpha}\left(-\mathfrak{B}_{11} \tau^{\alpha}\right)\right)}{\mathfrak{B}_{11}(t-\tau)^{\alpha+1} \Gamma(-\alpha)}+\mathfrak{B}_{10} E_{\alpha, \alpha}\left(-\mathfrak{B}_{11} \tau^{\alpha}\right)\right\} d \tau d \xi
$$

Equation (14) is an optimal and fractionalized solution of temperature analysis. The verification can also be obtained by $y=0$ and $t=0$ in Equation (14) to have the imposed conditions as defined in Equation (5).

\subsection{Use of Non-Singular Kernel for Momentum Equations}

Applying Laplace and Fourier Sine transforms on Equation (9) on Equation (7), we get

$$
\overline{u_{s}}(\xi, s)=\frac{\frac{\mathfrak{B}_{4}}{\mathfrak{B}_{0}} G_{r} \overline{\theta_{s}}(\xi, t) \cdot \cos \delta+\xi \sqrt{\frac{2}{\pi}} \frac{\mathfrak{B}_{1}}{\mathfrak{B}_{0}}\left(\frac{s\left(s^{\mathcal{q}}+\mathfrak{B}_{7}\right) \mathfrak{B}_{0} K}{s^{2}+\omega^{2}}\right)}{\left(s^{\mathcal{q}_{\mathfrak{B}_{12}}}+\mathfrak{B}_{13}\right)},
$$

Equation (15) is solved by letting variables defined as $\mathfrak{B}_{12}=\mathfrak{B}_{0} K \beta \lambda+\mathfrak{B}_{2} M K \sin \gamma+$ $\mathfrak{B}_{3} \beta+\mathfrak{\zeta}^{2} \mathfrak{B}_{1}, \mathfrak{B}_{13}=\mathfrak{B}_{2} M \sin \gamma \mathfrak{B}_{7} K \beta+\mathfrak{B}_{3} \beta \mathfrak{B}_{7}+\mathfrak{\zeta}^{2} \mathfrak{B}_{1} K \mathfrak{B}_{7}, \mathfrak{B}_{14}=\mathfrak{B}_{13}+\xi^{2} \mathfrak{B}_{1} \mathfrak{B}_{7} K / \mathfrak{B}_{12}+$ $\mathfrak{\zeta}^{2} \mathfrak{B}_{1} K$ and $\mathfrak{B}_{15}=\mathfrak{B}_{13} / \mathfrak{B}_{12}$. Equation (15) is invoked via inverse Fourier sine transform, the resultant expression is

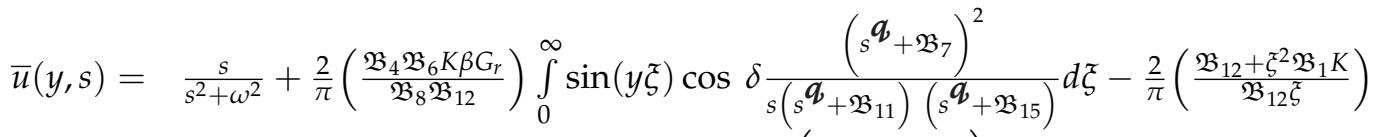

$$
\begin{aligned}
& \times \frac{s}{s^{2}+\omega^{2}} \int_{0}^{\infty} \sin (y \xi)\left(\frac{s^{\mathcal{q}}+\mathfrak{B}_{14}}{s\left(s^{\mathcal{q}}+\mathfrak{B}_{15}\right)}\right) d \xi
\end{aligned}
$$

Inverting Equation (16) by Laplace transform with special functions; see Appendix A (Equations (A8)-(A10)), the optimal solution of governing equation of momentum for Casson fluid with fractional derivative based on non-singular kernel is investigated as: 


$$
\begin{aligned}
& u(y, t)=\cos \omega t+\frac{2}{\pi}\left(\frac{\mathfrak{B}_{4} \mathfrak{B}_{6} K \beta G_{r}}{\mathfrak{B}_{8} \mathfrak{B}_{12}}\right) \int_{0}^{\infty} \sin (y \xi) \cos \delta \int_{0}^{t} \sum_{k=0}^{\infty} \sum_{m=0}^{\infty} \frac{(-1)^{k+m}\left(\mathfrak{B}_{11}\right)^{k+m} \tau^{\boldsymbol{q}_{k}}}{\Gamma\left(\boldsymbol{q}_{k+1}\right) \Gamma\left(\boldsymbol{q}_{m}\right)}(t-\tau)^{\boldsymbol{q}_{m}-1} d \tau d \xi \\
& +\frac{2}{\pi}\left(\frac{\mathfrak{B}_{4} \mathfrak{B}_{6} K \beta G_{r}}{\mathfrak{B}_{8} \mathfrak{B}_{12}}\right) \int_{0}^{\infty} \sin (y \xi) \cos \delta \int_{0}^{t} \sum_{k=0}^{\infty} \sum_{m=0}^{\infty} \frac{(-1)^{k+m}\left(\mathfrak{B}_{11}\right)^{k+m} \tau^{\boldsymbol{q}_{+} \boldsymbol{q}_{k}}}{\Gamma\left(\boldsymbol{q}_{+} \boldsymbol{q}_{k+1}\right) \Gamma\left(\boldsymbol{q}_{m}\right)}(t-\tau)^{\boldsymbol{q}_{m}-1} d \tau d \xi \\
& +\frac{2}{\pi}\left(\frac{\mathfrak{B}_{4} \mathfrak{B}_{6} K \beta G_{r}}{\mathfrak{B}_{8} \mathfrak{B}_{12}}\right) \int_{0}^{\infty} \sin (y \xi) \cos \delta \int_{0}^{t} \sum_{k=0}^{\infty} \sum_{m=0}^{\infty} \frac{(-1)^{k+m}\left(\mathfrak{B}_{11}\right)^{k+m} \tau^{2} \boldsymbol{q}_{+} \boldsymbol{q}_{k}}{\Gamma\left(2 \boldsymbol{q}_{+} \boldsymbol{q}_{k+1}\right) \Gamma\left(\boldsymbol{q}_{m}\right)}(t-\tau)^{\boldsymbol{q}_{m-1}} d \tau d \xi \\
& -\frac{2}{\pi}\left(\frac{\mathfrak{B}_{12}+\xi^{2} \mathfrak{B}_{1} K}{\mathfrak{B}_{12} \xi^{\xi}}\right) \int_{0}^{\infty} \sin (y \xi) \int_{0}^{t} \cos \omega(t-\tau) \mathrm{E}_{\boldsymbol{q}}\left(-\mathfrak{B}_{15} t^{\mathfrak{q}}\right) d \tau d \xi-\frac{2}{\pi} \frac{\mathfrak{B}_{14}}{\mathfrak{B}_{15}}\left(\frac{\mathfrak{B}_{12}+\xi^{2} \mathfrak{B}_{1} K}{\mathfrak{B}_{12} \xi}\right) \\
& \times \int_{0}^{\infty} \sin (y \xi) \int_{0}^{t} \cos \omega(t-\tau)\left(1-\mathrm{E}_{\boldsymbol{q}}\left(-\mathfrak{B}_{15} t^{\boldsymbol{q}}\right)\right) d \tau d \xi
\end{aligned}
$$

Equation (17) is an optimal and fractionalized solution of velocity profile. The verification can also be obtained by putting $y=0$ and $t=0$ in Equation (17) to have the imposed conditions as defined in Equation (5).

\section{Results with Parametric Discussion and Conclusions}

Due to high conductivity and high aspect ratio of nanotubes, fractionalized Casson fluid is developed for enhanced heat transfer based on suspension of ethylene glycol. The thermophysical properties of nanofluid for the temperature and velocity profiles have been investigated for giving the comparative analysis of single and multi-walled carbon nanotubes. The rheological parameters have been measured for the higher sensitivity, crystallinity, and functionalization of single and multi-walled carbon nanotubes to enhance heat transfer rate. In this regard, temperature distribution with ripple visualization verses non-ripple visualization for 2-D graphs subject to varying $\operatorname{Pr}$ have been observed in Figure 3 . It is perceived from the simulation code for testing the different Prandtl numbers Pr in which the momentum diffusivity dominates the behavior in both cases. Here, $\operatorname{Pr}>1$, so the typical response from ethylene glycol indicates that the heat conduction is more significant in comparison with convection, so thermal diffusivity is dominant. Temperature distribution with ripple visualizations for 3-D graphs showing spatial and time domain is sketched in Figure 4. A ripple visualization of Figure 4 is presented for the dependency of temperature distribution with respect to spatial and time domain. The influence of single and multiwalled carbon nanotubes on the temperature distribution is studied through comparison of single and multi-walled carbon nanotubes in Figure 5 for fractional differential parameter. It is observed that the functionalization of single and multi-walled carbon nanotubes is quite similar and identical when increase in fractional differential parameter occur. While, the dispersion and wettability of carbon nanotubes are noticed at smaller fractional differential parameter. The role of single and multi-walled carbon nanotubes for volume fraction on temperature distribution is sketched in Figure 6. The finding of the single and multi-walled carbon nanotubes for volume fraction has happened for physical aspect of nanofluid and the investigation of this innovative physical aspect has uninterruptedly been utilized for heat transfer of mechanical application. In short, enhancing trends for temperature is perceived with the increase in volume fraction. Physically, enhancing trends of temperature is due to increase of the addition of solid carbon nanotubes. To further corroborate the formation of single and multi-walled carbon nanotubes, the comparative analysis for temperature and velocity profiles for single and multi-walled carbon nanotubes is presented in Figure 7. Here, the single walled carbon nanotubes verse multi-walled carbon nanotubes in temperature distribution and velocity field have been inspected for checking the better performance. It is observed from Figure 7 that defectiveness of the multi-walled carbon nanotubes results the lowering profiles of temperature distribution and velocity field. The possible fractional and non-fractional mathematical models for velocity field are proposed to explain the formation of single and multi-walled carbon nanotubes through their comparison. Here four types of fractional and non-fractional mathematical models for velocity field have been discussed in Figure 8, namely (i) fractional velocity 
field with single walled carbon nanotubes, (ii) non-fractional velocity field with single walled carbon nanotubes, (iii) fractional velocity field with multi walled carbon nanotubes, and (iv) non-fractional velocity field with multi walled carbon nanotubes. It is observed from Figure 8 that fractional velocity field with single walled carbon nanotubes has larger velocity in comparison with other fractional and non-fractional mathematical models for velocity field. In light of above discussion, current study has investigated that fractional velocity field with single walled carbon nanotubes demonstrates the robustness of this new analysis. Additionally, this analysis can also be identical for temperature distribution.
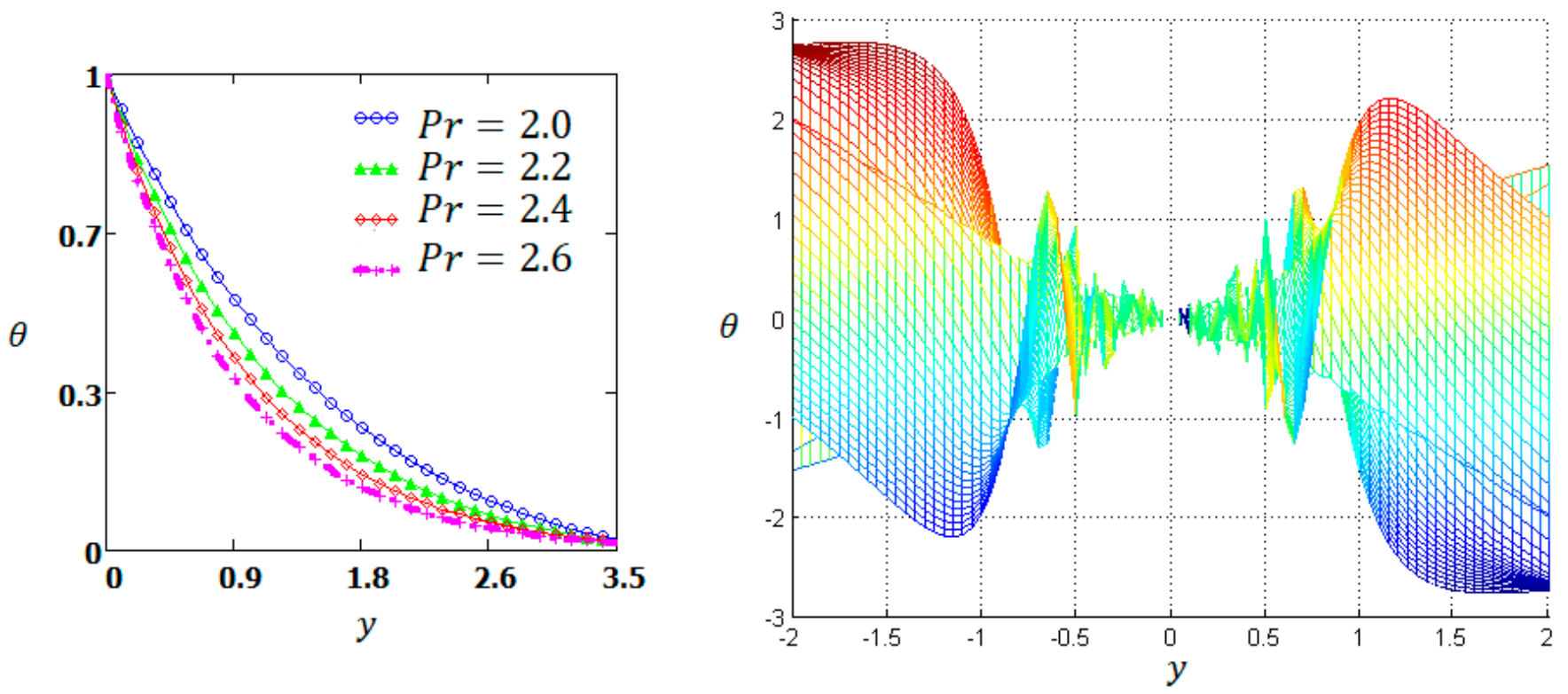

Figure 3. Temperature distribution with ripple visualization verses non-ripple visualization for 2-D graphs subject to varying Pr.
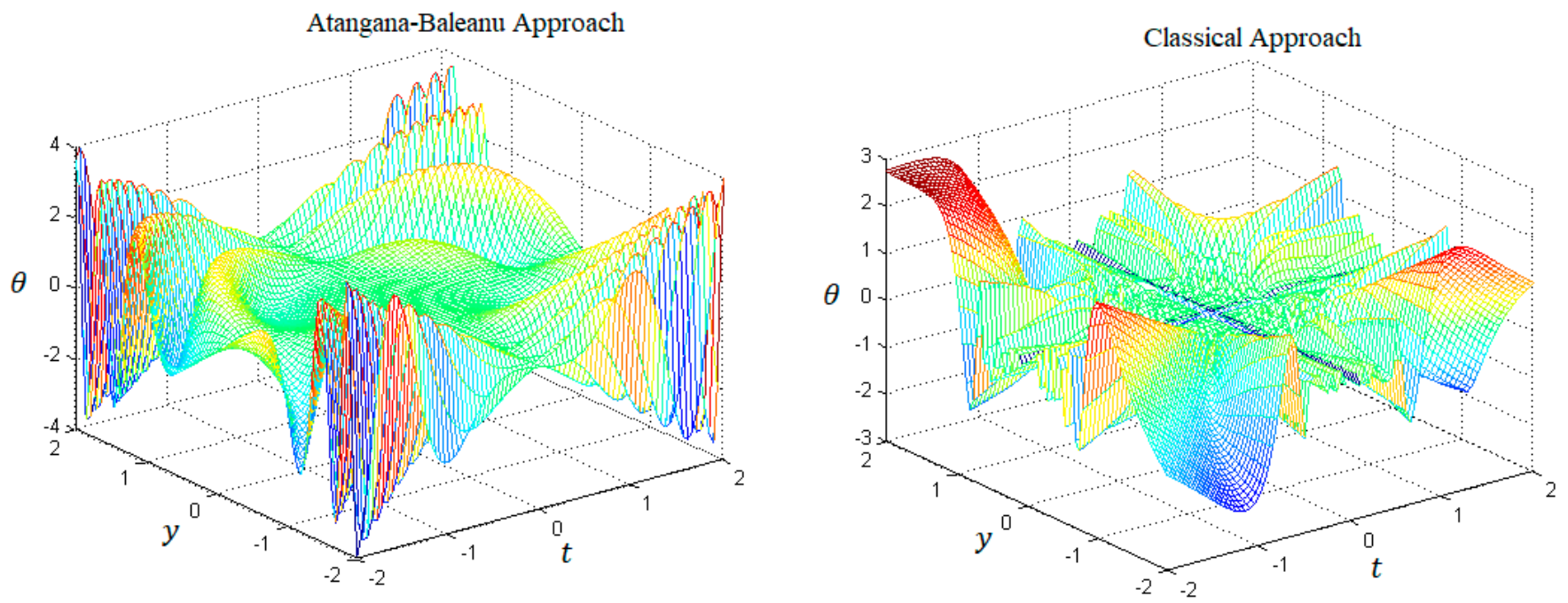

Figure 4. Temperature distribution with ripple visualizations for 3-D graphs showing spatial and time domain. 

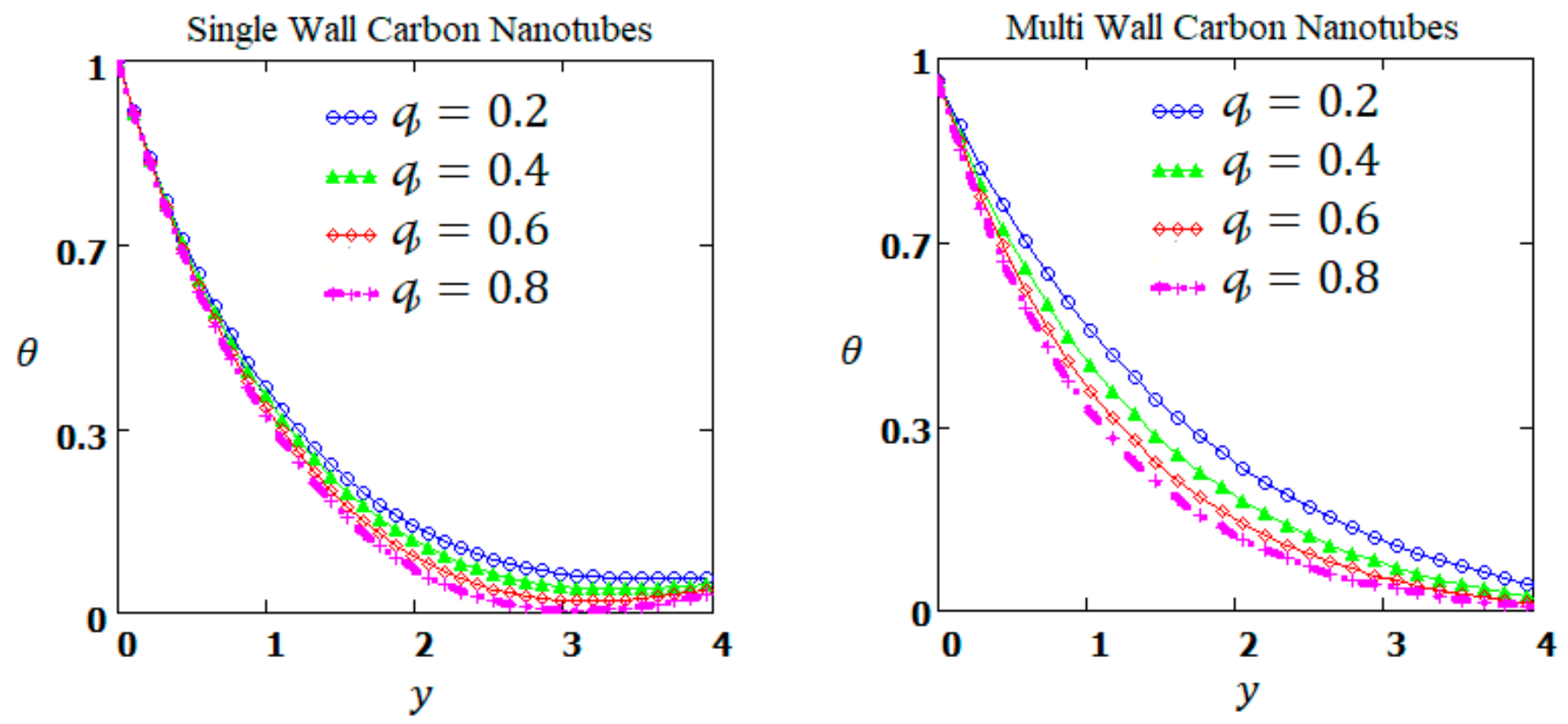

Figure 5. Temperature distribution of single and multi-wall carbon nanotubes without ripple visualizations for 2-D graphs subject to varying fractional parameter.
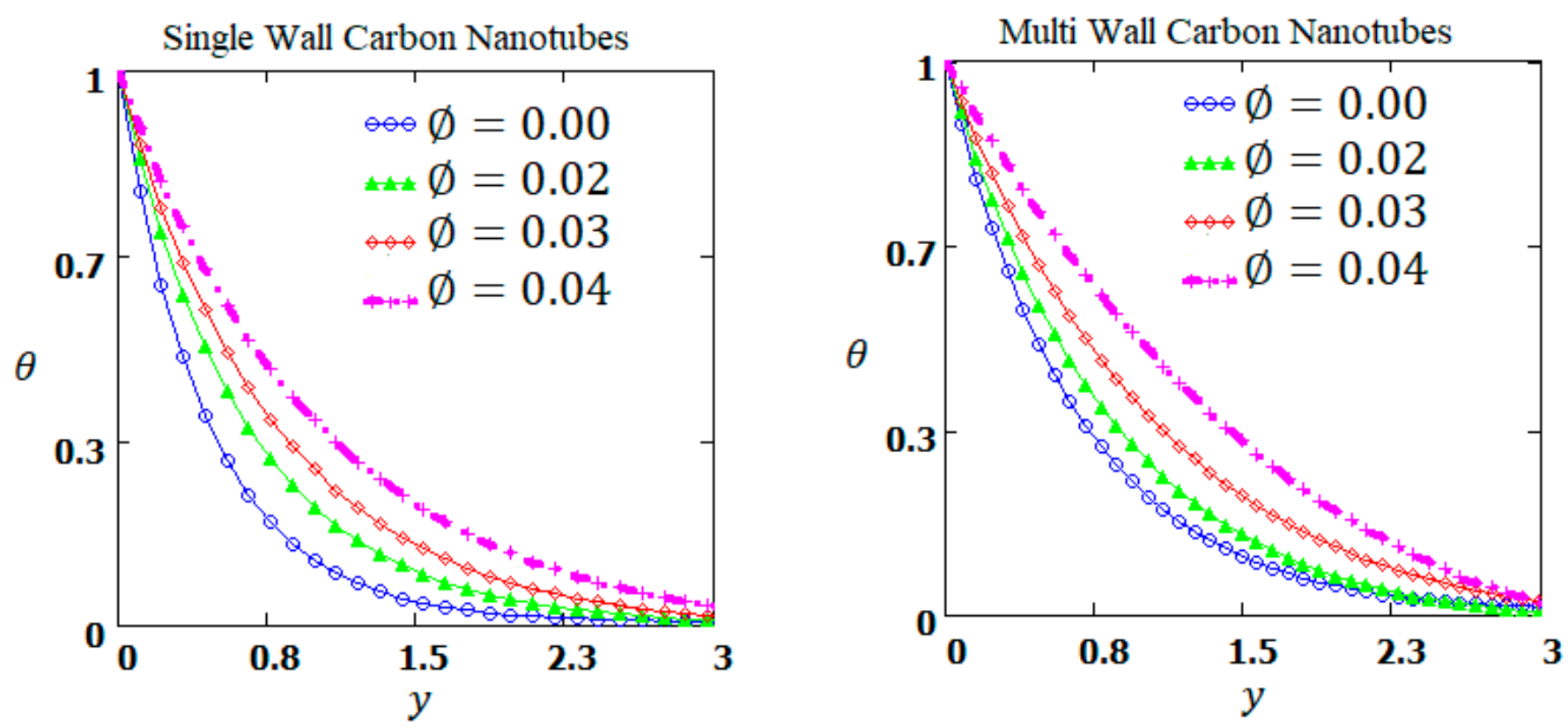

Figure 6. Temperature distribution of single and multi-wall carbon nanotubes without ripple visualizations for 2-D graphs subject to varying volume fraction. 
Single Wall Carbon Nanotubes

\section{Multi Wall Carbon Nanotubes}

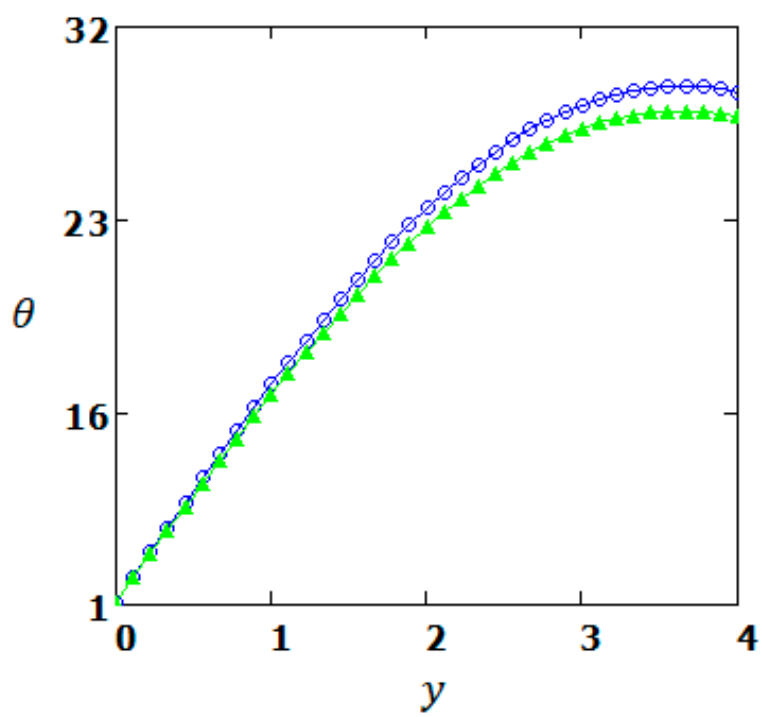

Single Wall Carbon Nanotubes

Multi Wall Carbon Nanotubes

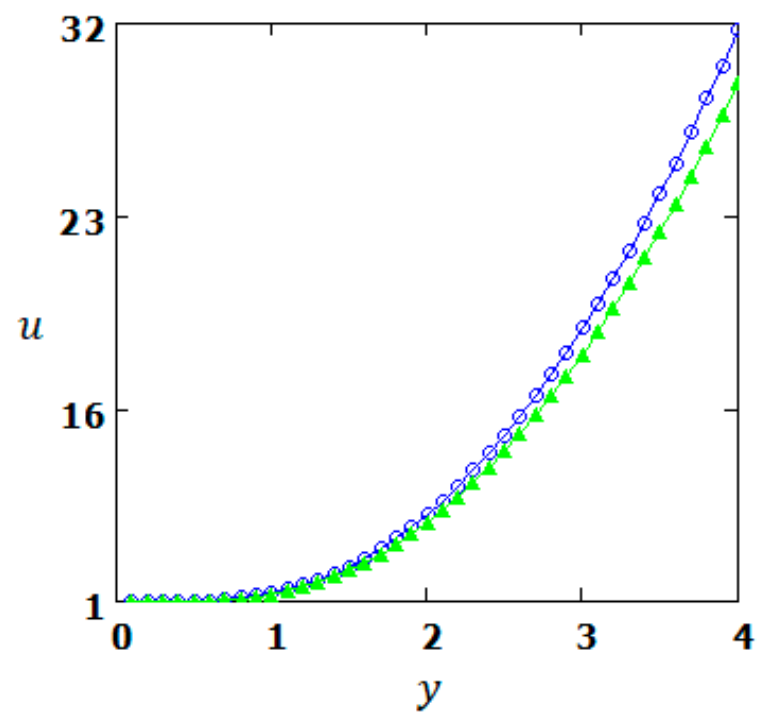

Figure 7. Temperature distribution and velocity profile of single and multi-wall carbon nanotubes without ripple visualizations for 2-D graphs.
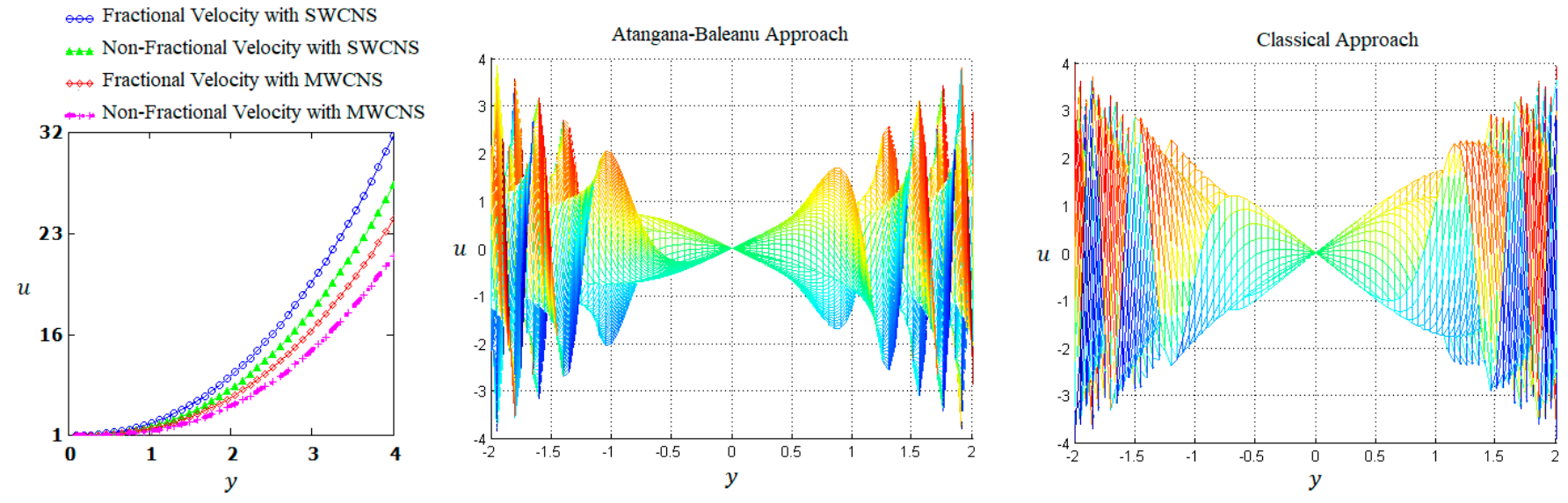

Figure 8. Velocity profile of single and multi-wall carbon nanotubes with and without ripple visualizations for 2-D and 3-D graphs based on fractional and non-fractional approaches.

\section{Conclusions}

An application of Fourier Sine transforms to carbon nanotubes suspended in ethylene glycol for the enhancement of heat transfer has been introduced in this work. We emphasized the generalized Mittage-Leffler kernel function is able to depict effective behaviors that has defined the complex nature of carbon nanotubes suspended in ethylene glycol for the enhancement of heat transfer. For the sake of simplicity, we encompassed the following outputs:

- The simulation for testing the different Prandtl numbers Pr dominates the behavior in both cases for the momentum diffusivity.

- A ripple visualization of temperature distribution presented the dependency of temperature distribution with respect to spatial and time domain.

- The comparison of single and multi-walled carbon nanotubes via fractional differential parameter observed the quite similar and identical dispersion and wettability of carbon nanotubes.

- The volume fraction on temperature distribution has enhancing trends for temperature. 
- The comparative analysis for temperature and velocity profiles for single and multiwalled carbon nanotubes in which defectiveness of the multi-walled carbon nanotubes results the lowering profiles of temperature distribution and velocity field.

- Fractional velocity field with single walled carbon nanotubes has larger velocity in comparison with other fractional and non-fractional velocity field models.

Author Contributions: Conceptualization, B.S. and K.A.A.; methodology, B.S. and K.A.A.; software, K.A.A.; validation, B.S., K.A.A. and H.A.; formal analysis, M.A.N.; investigation, B.S. and A.Y.; resources, K.A.A.; data curation, B.S.; writing—original draft preparation, K.A.A.; writing—review and editing, H.A and M.A.N.; visualization, B.S. and K.A.A.; supervision, K.A.A.; project administration, B.S.; funding acquisition, B.S. All authors have read and agreed to the published version of the manuscript.

Funding: This work was supported by the Deanship of Scientific Research, King Faisal University, Saudi Arabia, grant number NA000129.

Institutional Review Board Statement: Not applicable.

Informed Consent Statement: Not applicable.

Data Availability Statement: Data are available with corresponding author upon request.

Acknowledgments: The authors acknowledge the Deanship of Scientific Research, King Faisal University, Saudi Arabia for their financial support, grant number NA000129.

Conflicts of Interest: The authors declare no conflict of interest.

\section{Nomenclature}

$\begin{array}{llll}n f & \text { Nanofluid } & \rho_{n f} & \text { Density } \\ \mu_{n f} & \text { Dynamic viscosity } & \sigma_{n f} & \text { Electrical conductivity } \\ k_{n f} & \text { Thermal conductivity } & \beta_{T} & \text { Thermal expansion } \\ \left(\rho \beta_{T}\right)_{n f} & \text { Thermal expansion } & \left(\rho C_{p}\right)_{n f} & \text { Heat capacitance } \\ B_{0} & \text { Applied magnetic field } & \varnothing & \text { Porosity } \\ k & \text { Permeability } & T_{\infty} & \text { Ambient temperature of the plate } \\ T_{w} & \text { Wall temperature } & \gamma & \text { Inclined angle } \\ g & \text { Gravitational acceleration } & \mathfrak{B}_{1}-\mathfrak{B}_{5} & \text { Letting parameters } \\ \mathfrak{D}_{t}^{\mathfrak{q}} & \text { Atangana-Baleanu time-fractional } & & \\ & \text { differential operator } & & \end{array}$

\section{Appendix A}

$$
\begin{gathered}
\text { Density : } \rho_{n f}=(1-\varnothing) \rho_{f}+\varnothing \rho_{s,} \\
\text { Dynamic viscosity : } \mu_{n f}=\frac{\mu_{f}}{(1-\varnothing)^{2.5}}, \\
\text { Electric conductivity : } \sigma_{n f}=\left\{1+\frac{3\left(\frac{\sigma_{s}}{\sigma_{f}}-1\right) \varnothing}{\left(\frac{\sigma_{s}}{\sigma_{f}}+2\right)-\left(\frac{\sigma_{s}}{\sigma_{f}}-1\right) \varnothing}\right\} \sigma_{f}, \\
\text { Thermal expansion : }\left(\rho \beta_{T}\right)_{n f}=(1-\varnothing)\left(\rho \beta_{T}\right)_{f}+\varnothing\left(\rho \beta_{T}\right)_{s^{\prime}} \\
\text { Heat Capacitance : }\left(\rho C_{p}\right)_{n f}=(1-\varnothing)\left(\rho C_{p}\right)_{f}+\varnothing\left(\rho C_{p}\right)_{s^{\prime}} \\
\text { Thermal Conductivity : } \sigma_{n f}=\left\{\frac{(1-\varnothing)+2 \varnothing \frac{k_{s}}{k_{s}-k_{f}} \ln \frac{k_{s}-k_{f}}{2 k_{f}}}{(1-\varnothing)+2 \varnothing \frac{k_{s}}{k_{s}-k_{f}} \ln \frac{k_{s}+k_{f}}{2 k_{f}}}\right\} k_{f},
\end{gathered}
$$




$$
\begin{aligned}
& \beta=\frac{\beta_{0}}{1+\beta_{0}}, M=\frac{v_{f} \sigma_{f} B_{0}^{2}}{\rho_{f} U_{0}^{2}}, K=\frac{k U_{0}^{2}}{v_{f} \varphi}, \operatorname{Pr}=\left(\frac{\mu C_{p}}{k}\right)_{f}, \\
& G r=\frac{g\left(v \beta_{T}\right)\left(T_{w}-T_{\infty}\right)}{U_{0}^{3}}, \mathfrak{B}_{0}=\left(1-\varnothing_{n f}\right)+\mathfrak{B}_{\frac{\rho_{s}}{\rho_{f}}},
\end{aligned}
$$

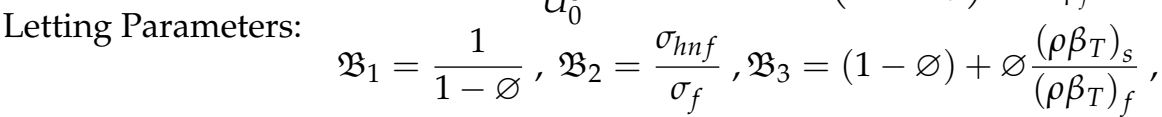

$$
\begin{aligned}
& \mathfrak{B}_{4}=(1-\varnothing)+\varnothing \frac{\left(\rho C_{p}\right)_{s}}{\left(\rho C_{p}\right)_{f}}, \mathfrak{B}_{5}=\frac{k_{n f}}{k_{f}}, \\
& \text { Dimensionless variables : } u^{*}=\frac{u}{U_{0}}, y^{*}=\frac{U_{0}}{v_{f}}, t^{*}=\frac{U_{0}^{2}}{v_{f}} t, \theta=\frac{T-T_{\infty}}{T_{\pi}-T_{\infty}} \text {, } \\
& {\left[\frac{1}{(x+a)}\right]=\sum_{k=0}^{\infty} \frac{(-x)^{k}}{a^{k+1}}} \\
& \mathcal{L}^{-1}\left[\frac{s^{\boldsymbol{q}}}{s\left(s^{\boldsymbol{q}}+\mathfrak{B}_{15}\right)}\right]=\mathrm{E}_{\boldsymbol{q}}\left(-\mathfrak{B}_{15} t^{\boldsymbol{q}}\right), \\
& \mathcal{L}^{-1}\left[\frac{\mathfrak{B}_{15}}{s\left(s^{\boldsymbol{q}}+\mathfrak{B}_{15}\right)}\right]=\left(1-\mathrm{E}_{\boldsymbol{q}}\left(-\mathfrak{B}_{15} t^{\boldsymbol{q}}\right)\right) \text {. }
\end{aligned}
$$

\section{References}

1. Hone, J.; Llaguno, M.C.; Biercuk, M.J.; Johnson, A.T.; Batlogg, B.; Benes, Z.; Fischer, J.E. Thermal properties of carbon nanotubes and nanotube-based material. Appl. Phys. A 2002, 74, 339-343. [CrossRef]

2. Xie, H.; Lee, H.; Choi, M. Nanofluids containing multiwall carbon nanotubes and their enhanced thermal conductivities. J. Appl. Phys. 2003, 94, 4967-4971. [CrossRef]

3. Abro, K.A.; Gomez-Aguilar, J.F. Role of Fourier sine transform on the dynamical model of tensioned carbon nanotubes with fractional operator. Math. Methods Appl. Sci. 2020, 1-11. [CrossRef]

4. Wen, D.; Ding, Y. Effective thermal conductivity of aqueous suspensions of carbon nanotubes. J. Thermophys. Heat Transf. 2004, 18, 481-485. [CrossRef]

5. Abro, K.A.; Laghari, M.H.; Gomez-Aguilar, J.F. Application of Atangana-Baleanu fractional derivative to carbon nanotubes based non-Newtonian nanofluid: Applications in nanotechnology. J. Appl. Comput. Mech. 2020, 6, 1260-1269. [CrossRef]

6. Halelfadl, S.; Estelle, P.; Aladag, B.; Doner, N.; Mare, T. Viscosity of carbon nanotubes water-based nanofluids: Influence of concentration and temperature. Int. J. Therm. Sci. 2013, 71,111-117. [CrossRef]

7. Halelfadi, S.; Mare, T.; Estelle, P. Efficiency of carbon nanotubes water based nanofluids as coolants. Exp. Therm. Fluid Sci. 2014, 53, 104-110. [CrossRef]

8. Kandasamy, R.; Mohamad, R.; Ismoen, M. Impact of chemical reaction on $\mathrm{Cu}, \mathrm{Al}_{2} \mathrm{O}_{3}$, and $\mathrm{SWCNTs-nanofluid} \mathrm{flow} \mathrm{under} \mathrm{slip}$ conditions. Eng. Sci. Technol. Int. J. 2016, 19, 700-709. [CrossRef]

9. Mustafa, M.; Junaid, K.A. Model for flow of Casson nanofluid past a non-linearly stretching sheet considering magnetic field effects. AIP Adv. 2015, 5, 077148. [CrossRef]

10. Kashif, A.A.; Ilyas, K. Effects of CNTs on magnetohydrodynamic flow of methanol based nanofluids via Atangana-Baleanu and Caputo-Fabrizio fractional derivatives. Therm. Sci. 2019, 23, 883-898. [CrossRef]

11. Kandasamy, R.; Muhaimin, I.; Mohammad, R. Single walled carbon nanotubes on MHD unsteady flow over a porous wedge with thermal radiation with variable stream conditions. Alex. Eng. J. 2016, 55, 275-285. [CrossRef]

12. Khan, I.; Saqib, M.; Alqahtani, A.M. Channel flow of fractionalized $\mathrm{H}_{2} \mathrm{O}$-based CNTs nanofluids with Newtonian heating. Discret. Contin. Dyn. Syst.-S 2019, 13, 769-779. [CrossRef]

13. Abro, K.A.; Jose, F.G.A. Fractional modeling of fin on non-Fourier heat conduction via modern fractional differential operators. Arab. J. Sci. Eng. 2021, 46, 2901-2910. [CrossRef]

14. Syed, T.S.; Kashif, A.A.; Sikandar, A. Role of single slip assumption on the viscoelastic liquid subject to non-integer differentiable operators. Math. Methods Appl. Sci. 2021, 44, 6005-6020. [CrossRef]

15. Kashif, A.A. Fractional characterization of fluid and synergistic effects of free convective flow in circular pipe through Hankel transform. Phys. Fluids 2020, 32, 123102. [CrossRef] 
16. Adesanya, S.O.; Souayeh, B.; Rahimi-Gorji, M.; Khan, M.N.; Adeyemi, O.G. Heat irreversibiility analysis for a couple stress fluid flow in an inclined channel with isothermal boundaries. J. Taiwan Inst. Chem. Eng. 2019, 101, 251-258. [CrossRef]

17. Abro, K.A.; Imran, Q.M.; Ambreen, S. Thermal transmittance and thermo-magnetization of unsteady free convection viscous fluid through non-singular differentiations. Phys. Scr. 2020, 96, 015215. [CrossRef]

18. Imran, Q.M.; Kashif, A.A.; Muhammad, A.S.; Asif, A.S. Functional shape effects of nanoparticles on nanofluid suspended in ethylene glycol through Mittage-Leffler approach. Phys. Scr. 2020, 96, 025005. [CrossRef]

19. Abro, K.A.; Mehwish, S.; Abdon, A.; Jose, F.G.A. Thermophysical properties of Maxwell Nanoluids via fractional derivatives with regular kernel. J. Therm. Anal. Calorim. 2020. [CrossRef]

20. Aziz, U.A.; Samia, R.; Samina, S.; Kashif, A.A. Fractional Modeling and Synchronization of Ferrouid on Free Convection Flow with Magnetolysis. Eur. Phys. J. Plus 2020, 135, 841-855. [CrossRef]

21. Qasim, A.; Samia, R.; Aziz, U.A.; Kashif, A.A. A mathematical model for thermography on viscous fluid based on damped thermal flux. Zeitschrift für Naturforschung A 2021, 76, 285-294. [CrossRef]

22. Qasim, A.; Samia, R.; Aziz, U.A.; Abro, K.A. Thermal investigation for electrified convection flow of Newtonian fluid subjected to damped thermal flux on a permeable medium. Phys. Scr. 2020, 95, 115003. [CrossRef]

23. Kashif, A.A. Role of fractal-fractional derivative on ferromagnetic fluid via fractal-Laplace transform: A first problem via fractal-fractional differential operator. Eur. J. Mech. B Fluids 2021, 85, 76-81. [CrossRef]

24. Muhammad, B.R.; Abro, K.A.; Khadijah MAbualnaja Akgul, A.; Aziz, U.R.; Muhammad, A.; Hamed, Y.S. Exact solutions involving special functions for unsteady convective flow of magnetohydrodynamic second grade fluid with ramped conditions. Adv. Differ. Equ. 2021, 2021, 408. [CrossRef]

25. Abro, K.A.; Atangana, A. A computational technique for thermal analysis in coaxial cylinder of one-dimensional flow of fractional Oldroyd-B nanofluid. Int. J. Ambient Energy 2021, 1-17. [CrossRef]

26. Aziz, U.A.; Mashal, A.; Naeem, U.; Sohail, N.; Kashif, A.A. Thermal analysis of oblique stagnation point low with slippage on second-order fluid. J. Therm. Anal. Calorim. 2021. [CrossRef]

27. Imran, S.; Shah, N.A.; Abro, K.A. Thermography of ferromagnetic Walter's-B fluid through varying thermal stratification. S. Afr J. Chem. Eng. 2021, 36, 118-126.

28. Saqib, M.; Kasim, A.R.M.; Mohammad, N.F.; Ching, D.L.C.; Shafie, S. Application of fractional derivative without singular and local kernel to enhanced heat transfer in CNTs nanofluid over an inclined plate. Symmetry 2020, 12, 768. [CrossRef]

29. Kashif, A.A.; Abdon, A. Synchronization via fractal-fractional differential operators on two-mass torsional vibration system consisting of motor and roller. J. Comput. Nonlinear Dyn. 2021. [CrossRef]

30. Abro, K.A.; Abdon, A. Strange Attractors and Optimal Analysis of Chaotic Systems based on Fractal-Fractional Differential Operators. Int. J. Model. Simul. 2021, 1-9. [CrossRef]

31. Atangana, A.; Baleanu, D. New fractional derivatives with nonlocal and non-singular kernel: Theory and application to heat transfer model. Therm. Sci. 2016, 20, 763-769. [CrossRef]

32. Kashif, A.A. Numerical study and chaotic oscillations for aerodynamic model of wind turbine via fractal and fractional differential operators. Numer. Methods Part. Differ. Equ. 2020, 1-15. [CrossRef]

33. Ali, K.A.; Atangana, A. Numerical and mathematical analysis of induction motor by means of AB-fractal-fractional differentiation actuated by drilling system. Numer Methods Part. Differ. Equ. 2020, 1-15. [CrossRef]

34. Ullah, A.; Selim, M.M.; Abdeljawad, T.; Ayaz, M.; Mlaiki, N.; Ghafoor, A. A magnetite-water-based nanofluid three-dimensional thin film flow on an inclined rotating surface with non-linear thermal radiations and couple stress effects. Energies 2021, 14, 5531. [CrossRef]

35. Mostafizur, R.M.; Rasul, M.G.; Nabi, M.N. Energy and exergy analyses of a flat plate solar collector using various nanofluids: An analytical approach. Energies 2021, 14, 4305. [CrossRef]

36. Kashif, A.A.; Abdon, A.; Jose, F.G.A. An analytic study of bioheat transfer Pennes model via modern non-integers differential techniques. Eur. Phys. J. Plus 2021, 136, 1144. [CrossRef] 